\title{
Molecular dynamics simulation of thermal and mechanical properties of polyimide-carbon-nanotube composites
}

\author{
Dewei $\mathrm{Qi}^{1}$, Jeffrey Hinkley ${ }^{2}$ and Gouwei $\mathrm{He}^{3}$ \\ ${ }^{1}$ Department of Paper Engineering, Chemical Engineering and Imaging, College of Engineering \\ and Applied Science, Western Michigan University, Kalamazoo, MI 49008, USA \\ 2 Advanced Materials and Processing Branch, NASA Langley Research Center, MS 226, \\ 6A West Taylor Street, Hampton, VA 23681, USA \\ ${ }^{3}$ Laboratory for Nonlinear Mechanics, Institute of Mechanics, Chinese Academy of Sciences, \\ Beijing 100080, People's Republic of China
}

Received 1 September 2004, in final form 8 February 2005

Published 8 April 2005

Online at stacks.iop.org/MSMSE/13/493

\begin{abstract}
An aromatic polyimide and its mixture with randomly distributed carbon nanotubes (NTs) are simulated by using molecular dynamics, repeated energy minimization and cooling processes. The glass transition temperatures are identified through volume-temperature curves. Stress-strain curves, Young's moduli, densities and Poisson ratios are computed at different temperatures. It is demonstrated that the carbon NT reduces the softening effects of temperature on mechanical properties and increases the ability to resist deformation.
\end{abstract}

\section{Introduction}

Nano-composites are rapidly growing research areas. A small amount of fillers could be mixed with polymers to achieve novel properties by combining properties from the parent constituents in a single material. There is also the possibility of new properties which are unknown in the parent constituent materials.

Single clay layers were proposed as an ideal reinforcing agent in 1997 [1] due to their extremely high aspect ratio and their thickness, comparable with the scale of the polymer chain. Later, the Toyota research group had a major breakthrough in polymer/clay nano-composite technology [2]. Their method of synthesis of nylon 6/clay nano-composites is economically suitable for industrial production. It was found that a doubling of both the tensile modulus and strength was achieved with as little as 4.7 weight per cent clay due to the enhanced interaction between the large surface areas of nanoscale clay fillers and polymers. Over the last decade, polymer/clay nano-composites have been observed to exhibit a dramatic enhancement in mechanical properties, barrier, flammability resistance and ablation performance. The efficiency of enhancement depends on the filler properties, size, aspect ratio and orientation and the adhesion between the fillers and the polymer matrix. Various polymer systems were used 
as the matrix including polyimides, elastomers and epoxies. Different levels of enhancement are achieved, depending on the chosen matrix polymers and fillers.

Since the discovery of carbon nanotubes (NTs), they have attracted tremendous attention. It was found that the mechanical, chemical, electrical, optical, magnetic and electro- and magneto-optical properties of these NTs are different from their bulk properties due to the quantum confinement effects. For example, theoretical and experimental results reveal that the Young's modulus of carbon NTs could be as high as 1-5 TPa [3] and the tensile strength could be as high as $200 \mathrm{GPa}$ [4]. Moreover, their aspect ratio is very high and the diameter scale is comparable with polymer chains. Thus, carbon NTs naturally suggest themselves for use as fillers to enhance the mechanical properties of composites. Indeed, Park et al [5] experimentally studied the mechanical properties of polyimide/single wall carbon NT composites. They found that the storage modulus increased by $65 \%$ upon addition of $1 \%$ carbon NTs by volume. Frankland et al [6] simulated the stress-strain behaviour of polyethylene-NT composites by using molecular dynamics (MD) simulations and found that long carbon NTs, but not short ones, could reinforce mechanical properties. Carbon NT composites appear to be promising materials with extremely high mechanical strength and very light weight. Further studies on these materials are necessary.

In this work, atomistic modelling will be used to investigate temperature effects on the stress-strain behaviour of the aromatic copolyimide (LARC-SI) resin and its composites with single-walled carbon NTs. We will focus on numerical simulations of the performance of carbon NTs employed as fillers in a polymeric material and on the evaluation of its improvement with respect to mechanical properties. Several MD simulations were conducted to study how carbon NTs affect the mechanical properties of polyimide composites and how temperature affects the tensile-strain curves of polyimide-NT composites.

Since polymers that contain aromatic rings tend to give better mechanical and thermal properties and good environmental resistance, the wholly aromatic thermoplastic copolyimide LARC-SI is chosen as a composite matrix. This polyimide may have high adhesive strength with carbon NTs [7,8].

Section 2 briefly describes the simulation method. This section also expresses the method of construction of NT crystals, LARC-SI polymer composites and polyimide-carbon-NT composites. Section 3 gives the results and a discussion. The final section summarizes our conclusions.

\section{Simulation method}

Generally speaking, there are two methods that can be used to simulate the mechanical properties. One is called molecular mechanics [9]. In this method, the potential energy of the system is minimized and the stresses are computed after the strain is applied. Essentially, the energy minimized structure corresponds to a system at $0 \mathrm{~K}$. Therefore, this method cannot be used to examine temperature effects. Another method is MD simulations. The MD method can be used to investigate how physical properties vary with temperature and time. Since the molecular structure of LARC-SI is much more complex than polyethylene, special efforts are required to construct LARC-SI polymers.

MD simulations were used to model the mechanical properties of carbon NT polymeric composites. A parallel code called LAMMPS [10] was employed. The code was slightly modified by the present authors to add some subroutines for the Tripos 5.2 force field. The Tripos 5.2 force field is well known [11] and its validity for polyimides and aromatic polymers that have five- and six-membered ring structures has been extensively demonstrated [12]. The 


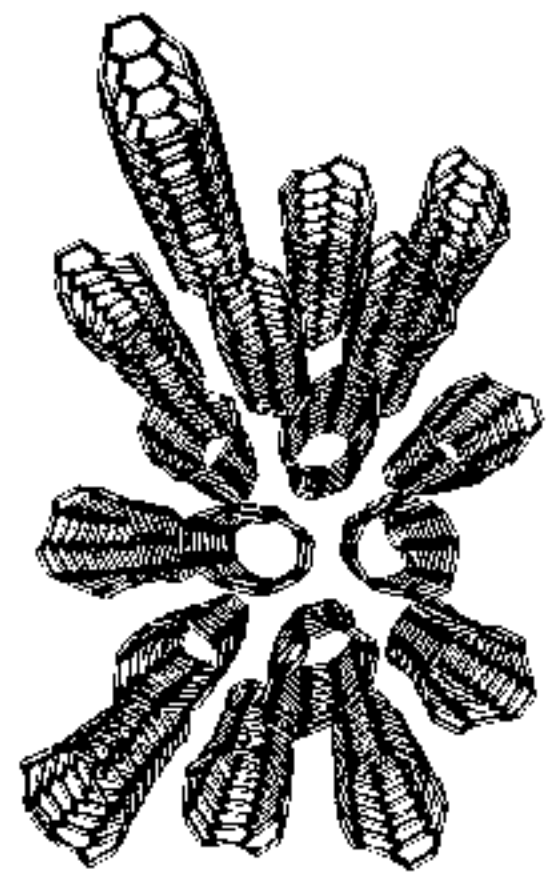

Figure 1. (4, 4) Carbon NT crystal with 18 tubes in the simulation box at $T=293 \mathrm{~K}$.

formulae for $L-J$ nonbonding, bonding, bending, torsion and out of plane potential terms and all parameters are given in [11] and will not be repeated here.

In the following sections, we will construct models of carbon NT crystals, LARC-SI polymers and polymer/single-walled carbon NT mixtures.

\subsection{Carbon NT crystal}

In this section, we construct a model of a carbon NT crystal for the purposes of evaluating the applicability of the Tripos force field to small carbon NTs. As illustrated in figure 1, the axes of the tubes are set along the $z$-direction of a simulation box and the tubes form a triangular close-packed crystal lattice in the $x-y$ plane with a lattice constant $2 r+l$, where $r$ is the tube radius and $l$ is the intertube separation. The radius of each single-walled tube is $r=2.71 \AA$, and $l=3.15 \AA$ initially. These are $(4,4)$ tubes, where the $(n, m)$ notation refers to the chiral vector of the NT in terms of the primitive in-plane lattice vectors of a graphene sheet [13]. There are 18 carbon NTs and 3024 atoms in the simulation box. Periodic boundary conditions are imposed for all the MD runs in this work.

Bonds in the NT are described using aromatic carbon-carbon bonding parameters in the Tripos 5.2 force field, but with the equilibrium bonding distance in the NT adjusted to its experimental value of $1.42 \AA$.

MD simulations were run at pressure $p=1 \mathrm{~atm}$ and temperature $T=293 \mathrm{~K}$ for $120 \mathrm{ps}$ using the Nose-Hoover algorithm $[14,15]$. The time step was $1 \mathrm{fs}$ and the cut-off distance for nonbonded interactions in the crystal was $10 \AA$. Then the system was cooled from $T=293$ to $T=10 \mathrm{~K}$ by scaling velocities at a constant cooling rate of $-1.96 \mathrm{~K}(\mathrm{ps})^{-1}$. After the temperature reached at $T=10 \mathrm{~K}$, the system was run for $30 \mathrm{ps}$. Next, the average dimensions of the simulation box were measured over another 30 ps. Subsequently, the crystal was stretched 


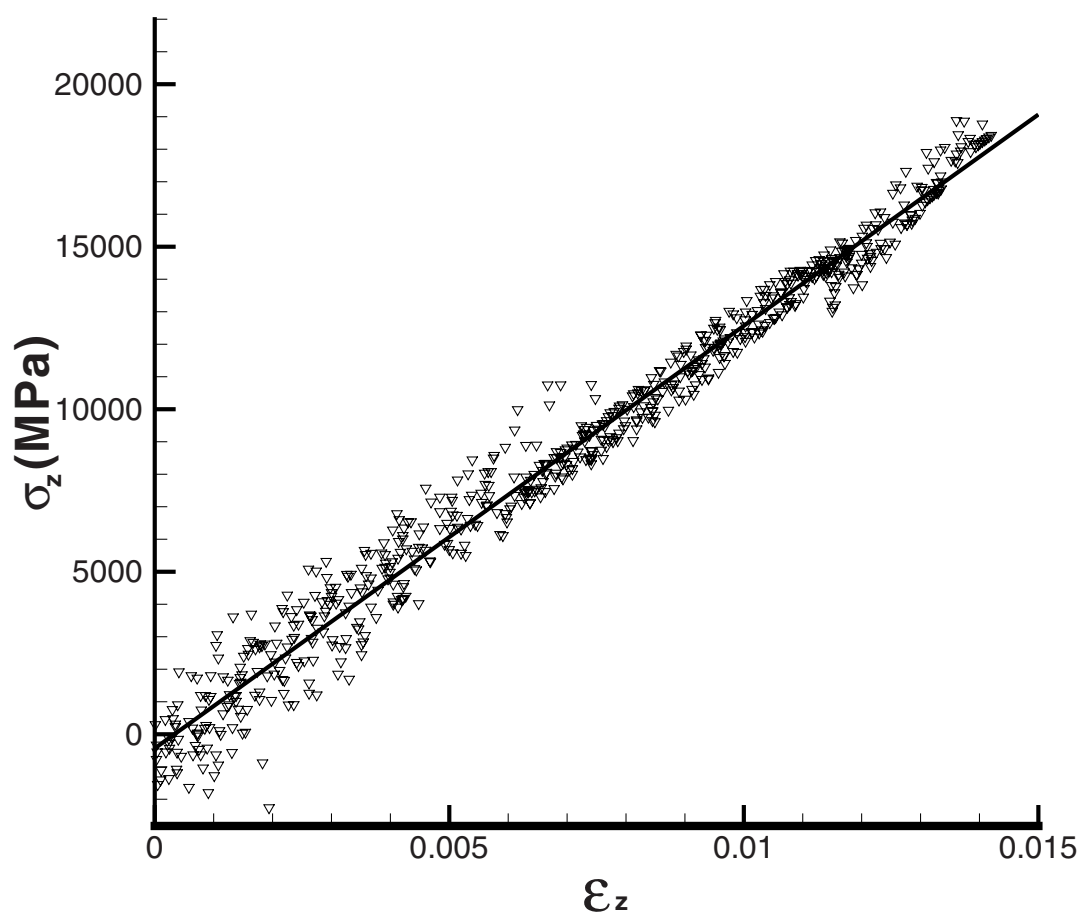

Figure 2. The stress in the NT axis direction as a function of strain for NT crystal at $T=10 \mathrm{~K}$. Each point is an average value over a $1 \mathrm{ps}$ time internal.

at $T=10 \mathrm{~K}$ by decreasing the external applied pressure in the $z$-direction or tube axis-direction at a constant rate $\alpha_{z}=\Delta p / \Delta t=-250 \mathrm{~atm}(\mathrm{ps})^{-1}$. Here $\Delta p$ is the pressure change and $\Delta t$ the stretching time increment. The external pressure was held constant at $p=1 \mathrm{~atm}$ in the $x$ - and $y$-directions.

The stress in response to the external tension is measured during stretching by

$$
\sigma=\frac{1}{V} \sum_{i}\left(\frac{m_{i}}{2} v_{i} v_{i}+F_{i} r_{i}\right)
$$

where $V$ is the volume; $v_{i}$ is velocity; $m_{i}$ is the mass; $r_{i}$ is the position; and $F$ is the force on the $i$ th particle. The stresses and dimensional changes of the simulation box are recorded during stretching. Young's modulus could be calculated as the initial slope of the stress-strain curve. This method was first used by Brown and Clarke [16], and a similar method was also reviewed by Ray [17].

The calculated stress, $\sigma_{z}$, as a function of strain, $\varepsilon_{z}$, is plotted in figure 2. Each point in the figure is an average value over a 1 ps time interval. From the slope of the linear fit, we obtain Young's modulus $E_{z}=1.30 \mathrm{TPa}$ for this $(4,4)$ crystal. The same method is applied to a $(10,10)$ crystal and yields a Young's modulus $E_{z}=0.89 \mathrm{TPa}$.

Laboratory measurements of the amplitude of the intrinsic thermal vibrations of the tubes yield an average value of 1.8 TPa with a very large variation [18]. Experimental bending measurements on the Young's modulus give an average value of $1.28 \pm 0.59 \mathrm{TPa}$, while recent tight-binding calculations produce a value of the order of $1 \mathrm{TPa}$ [19]. It is clear therefore that our calculated Young's moduli are in the same range as other authors'. In addition, Young's modulus decreases as the radius of the tube increases [20]. The $(4,4)$ and $(10,10)$ tube results 


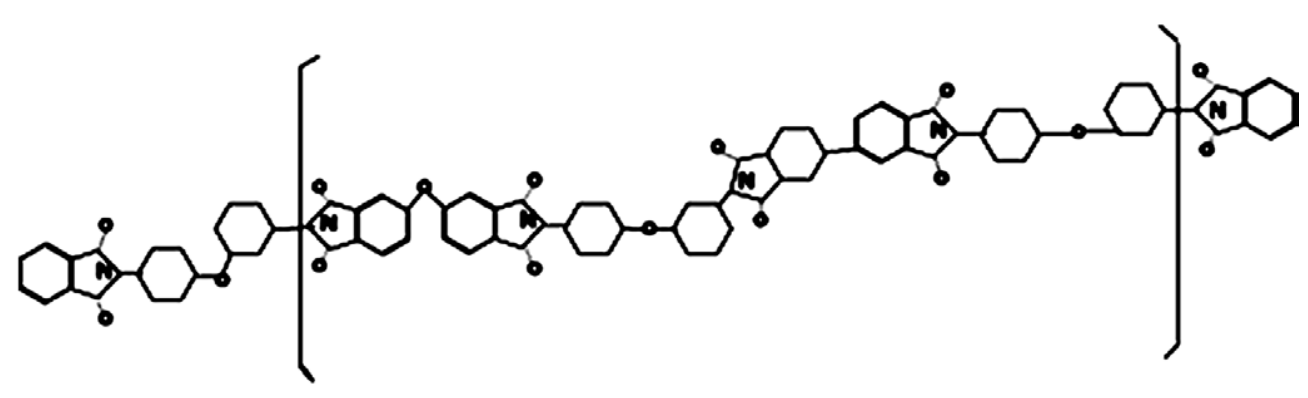

Figure 3. Chemical structure of LARC-SI polyimide.

indicate that the chosen force field correctly captured this trend. In the following sections, the construction of the polymer assembly and two NT composites is described.

\subsection{Polyimide}

One basic repeat unit of the LARC-SI polymer is illustrated in figure 3 . The $50: 50$ copolymer is modelled as strictly alternating, which is probably a simplification. The asymmetric diamine $3,4^{\prime}$ oxydianiline can insert in the chain in two directions; this minor structural detail is ignored.

Since amorphous polymers have a more complex structure than crystals, we have developed a variation of a Monte Carlo method [21] that we call POLY-PIC to construct the amorphous assembly. In our code, planar five- and six-membered rings with equilibrium atomic positions and angles are pre-constructed in a body-fixed coordinate system. Three randomly-chosen Euler angles control the orientation in which the successive rings are added to the molecular chain, and the coordinates are transformed to a space-fixed coordinate system. As each ring or connecting atom is connected, nonbonded distances are checked. If the distance between any two atoms is less than a pre-set value, say $1.8 \AA$, we delete the added ring or atom and assign new random numbers to this ring or atom. If the distance is still smaller than the pre-set value after many iterations, then the entire molecule is deleted and reconstructed. In the polyimide structure, illustrated in figure 4, there are eight LARC-SI molecules. Each molecule has six repeated units (645 atoms). The total number of atoms is 5160 .

We initially construct a polyimide melt with a density of $\rho=0.688 \mathrm{~g} \mathrm{~cm}^{-3}$, and adopt the view, a well-known concept for $L-J$ glasses, that solid amorphous materials after cooling from their liquid state will keep the same structure as the liquid state.

\subsection{Composites}

The first composite structure consists of seven LARC-SI polyimide molecules and one carbon NT. The carbon NT with 546 atoms is first placed along the $z$-direction in the centre of the simulation box. With the periodic boundaries, this corresponds to an infinitely long tube. Next seven LARC-SI molecules are randomly distributed by using POLY-PIC. Each polymer chain in the composite is the same as that in the polyimide box. The total number of atoms in this model composite is 5061, and the NT comprises $16 \%$ of the weight. The initial density is $\rho=0.695 \mathrm{~g} \mathrm{~cm}^{-3}$. A snapshot of this composite is illustrated in figure 5 .

The third simulation contains ten short carbon NTs mixed with 25 LARC-SI polyimide molecules as illustrated in figure 6. POLY-PIC constructs the composite by randomly either adding an NT or constructing a molecule. Each $(4,4)$ carbon NT has 616 atoms and is $95.86 \AA$ long; each LARC-SI polyimide molecule is the same as those in the first and second structures. 


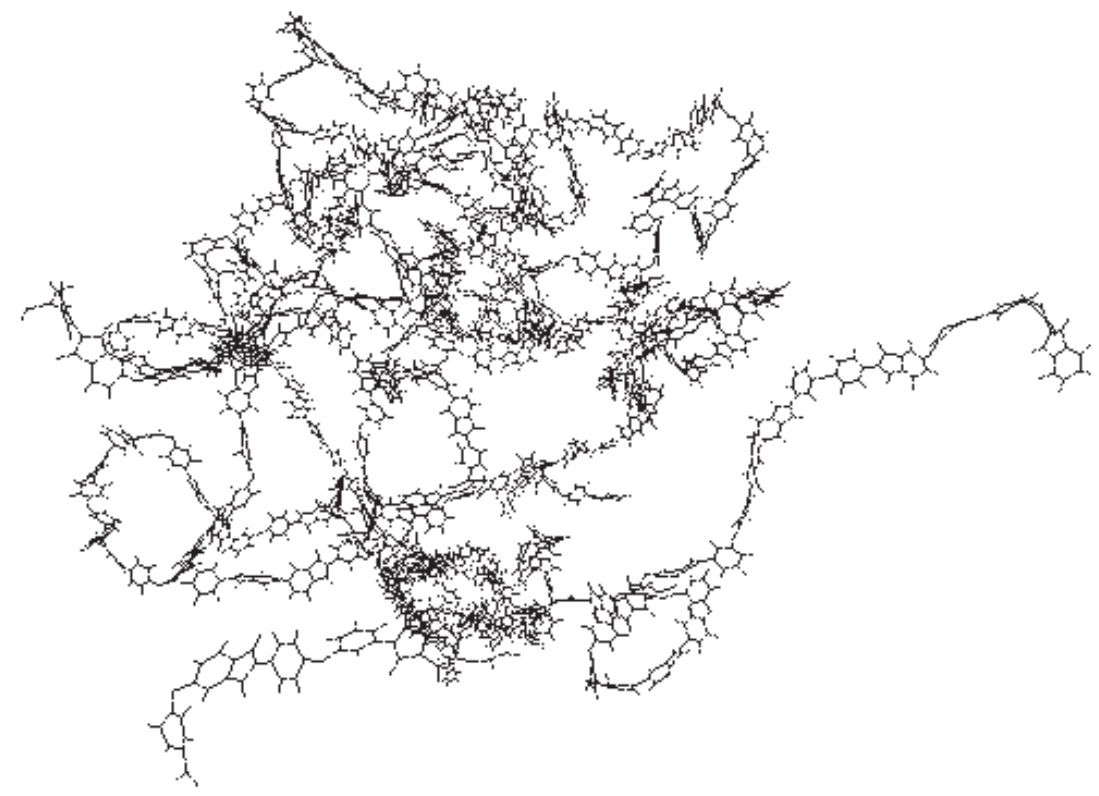

Figure 4. An assembly of eight LARC-SI polymer chains. The simulation box is not shown.

The initial density is $0.354 \mathrm{~g} \mathrm{~m}^{-3}$, the total number of atoms is 22285 and the NTs are $33 \%$ by weight. We call this a short-tube composite.

From the initial configurations described above, we run constant pressure MD simulations at $p=1 \mathrm{~atm}$ and $T=1000 \mathrm{~K}$. The cut-off distance for unbonded interactions for composites is $15 \AA$. Essentially, each system is compressed from its initial size to its equilibrium size.

Following the work of Fan and Hsu [9] and other authors [22,23], we use the MD simulation and repeated energy minimization to create correct amorphous structures. The initial temperature $T=1000 \mathrm{~K}$ is far above the glass transition temperature of LARC-SI $\left(T_{\mathrm{g}}=522 \mathrm{~K}[24]\right)$ to ensure a random melt structure. Each structure is initially relaxed for a total of at least $150 \mathrm{ps}$, and then we alternately minimize the energy using a Hessian-free truncated Newton method and perform 60 ps MD runs. There are six minimization/MD cycles, in total, to ensure a relaxed structure.

Next, the composites are cooled from $T=1000 \mathrm{~K}$ to lower temperatures at a constant cooling rate of $-1.96 \mathrm{~K}(\mathrm{ps})^{-1}$. Configurations at temperatures of interest are stored during the cooling process. Each of the stored configurations is then run for $30 \mathrm{ps}$ at the corresponding temperature and $p=1 \mathrm{~atm}$ to reach an equilibrium state. During MD runs we relax the simulation box size in the $x$-, $y$ - and $z$-directions independently without tension coupling. Therefore, although the initial simulation box is a cube, the final simulation box lengths in the three directions may be different. The final density at room temperature $T=293 \mathrm{~K}$ was $1.21 \mathrm{~g} \mathrm{~cm}^{-3}$ for the polyimide composite, $1.23 \mathrm{~g} \mathrm{~cm}^{-3}$ for the infinitely-long-tube composite and $1.35 \mathrm{~g} \mathrm{~cm}^{-3}$ for the short-tube composite.

\section{Simulation results and discussion}

For each composite, after repeated energy minimizations and MD runs, we obtained a well relaxed amorphous structure. A radial distribution function (RDF), $g(r)$, for all atoms of 


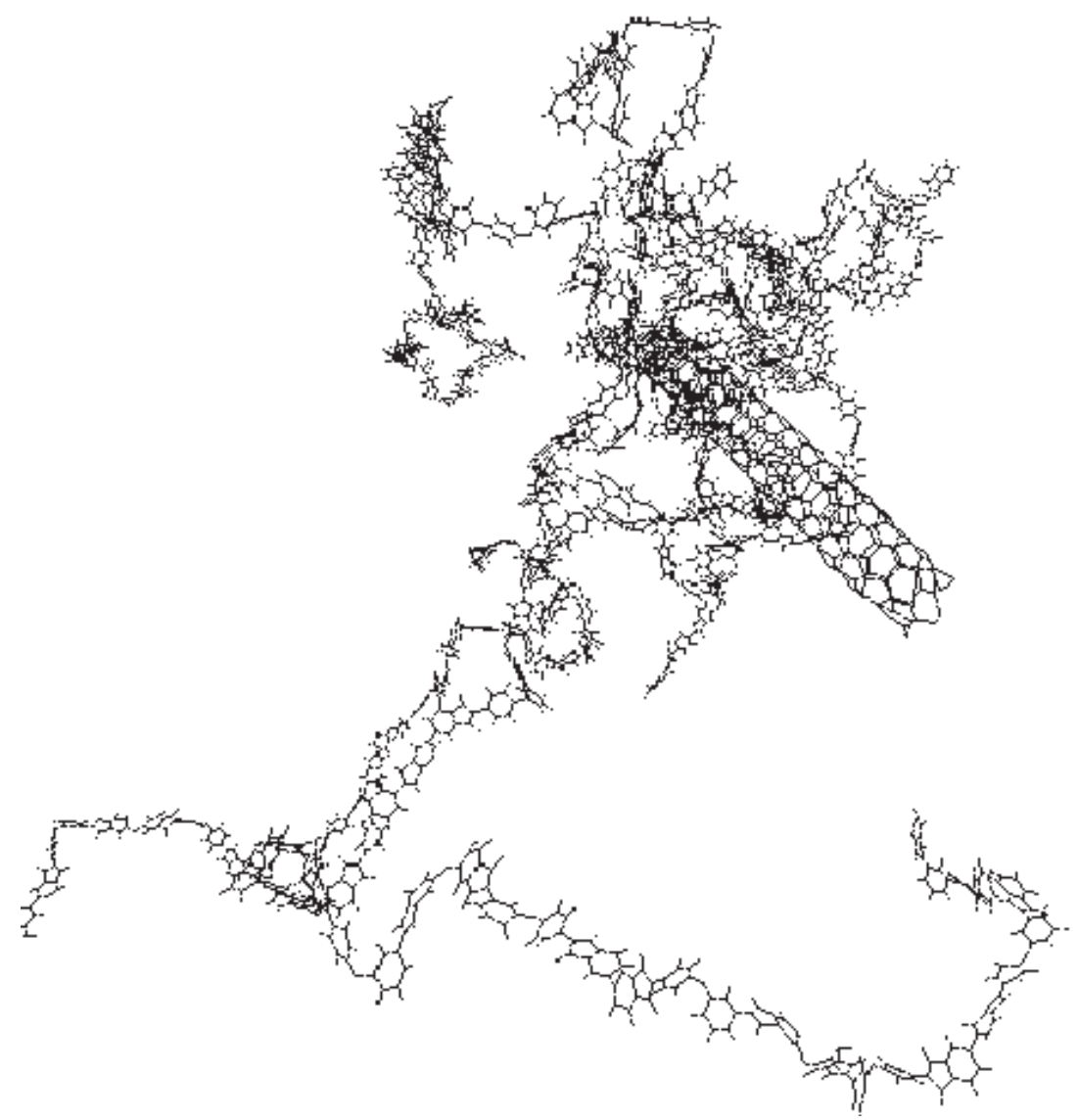

Figure 5. Polyimide-infinitely-long-tube composite consisting of seven LARC-SI polymer chains and a single infinitely-long NT.

LARC-SI at $T=293$ is plotted in figure 7. The first peak in the RDF corresponds to the distance between hydrogen and carbon atoms and the second peak corresponds to the distance between carbon atoms in the rings. When $r>5 \AA$, the RDF is flat and approaches 1.0, demonstrating that the material lacks long range order; i.e. it is amorphous.

The results for the density as a function of temperature are plotted in figure 8 for both the polyimide and the polyimide-infinitely-long-tube composite. The density at constant pressure $p=1 \mathrm{~atm}$ is an average over $30 \mathrm{ps}$ at each temperature. There are two portions that could be linearly fitted in each curve. It is clear that the slope in the solid region is smaller for the polyimide-infinitely-long-tube composite than for the polyimide. In other words, the volume thermal expansion coefficient has been reduced by adding the carbon NTs.

It is well known that for an amorphous $L-J$ system, a glass transition can be identified from the density-temperature curve. We apply this knowledge to MD simulations of our polymer systems. We observe that the slope of the line above $T=570 \mathrm{~K}$ is larger than that below $T=570 \mathrm{~K}$. The intersection of the two lines gives a glass transition temperature of $T_{\mathrm{g}}=570 \mathrm{~K}$ for the polyimide, about $40 \mathrm{~K}$ higher than the experimental result $[7,24]$. That it is higher is not surprising because we have used a very high cooling rate, and a higher cooling rate leads to a higher glass transition temperature. For the polyimide-infinitely-long-tube composite, $T_{\mathrm{g}}=550 \mathrm{~K}$. 


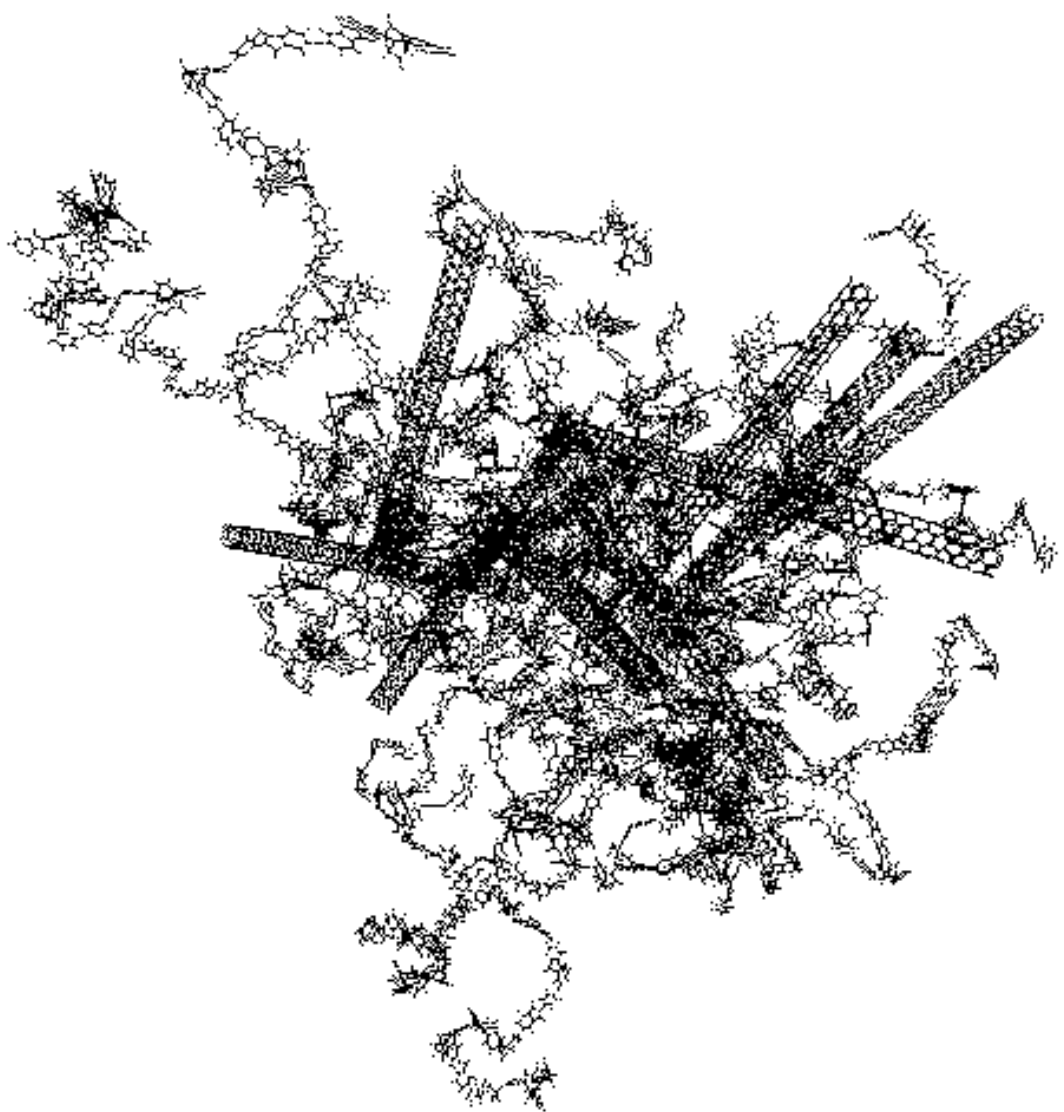

Figure 6. The polyimide-short-tube composite consists of 25 LARC-SI polymer chains and ten NTs.

Simulated stress-strain curves in the $z$-direction are plotted in figure 9 for the polyimide assembly and in figure 10 for the short-tube composite. The stress-strain curves in each of the three directions are similar. (The curves in other directions are not shown.) To reduce the effects of fluctuations, we obtain each point in these figures by averaging MD results of stresses and strains over 1 ps time intervals during stretching.

It is seen that for both the un-reinforced polyimide and the short-tube composite the curves deviate from linearity around strain $\varepsilon_{z}=0.08$. The Young's modulus is obtained by a linear fit to the portion of the strain-stress curve up to a strain of $\varepsilon=0.04-0.05$. Both the initial slopes and the plastic properties (nonlinear portions) vary with temperature.

Since the stress-strain curves and Young's modulus may depend on the stretching or tension rates, the effects of tension rates on Young's modulus were examined. Stress-strain curves for the polyimide at two different tension rates are shown in figure 11. The higher tension rate $\alpha_{z}=-250$ atm (ps) ${ }^{-1}$ is 16.66 times larger than the lower tension rate, while the Young's modulus at $T=293 \mathrm{~K}$, calculated from the linear portion of the stress-strain curve, is only $16.36 \%$ larger than at the lower tension rate. In other words, a 16.66 times difference in the tension ratio results in only a $16 \%$ difference in the Young's modulus ratio, indicating the Young's modulus is not very sensitive to the strain rate.

In this work we are most interested in how mechanical properties are affected by carbon NTs - that is, in relative increases, not in the absolute values. The effect of the tension rate is 


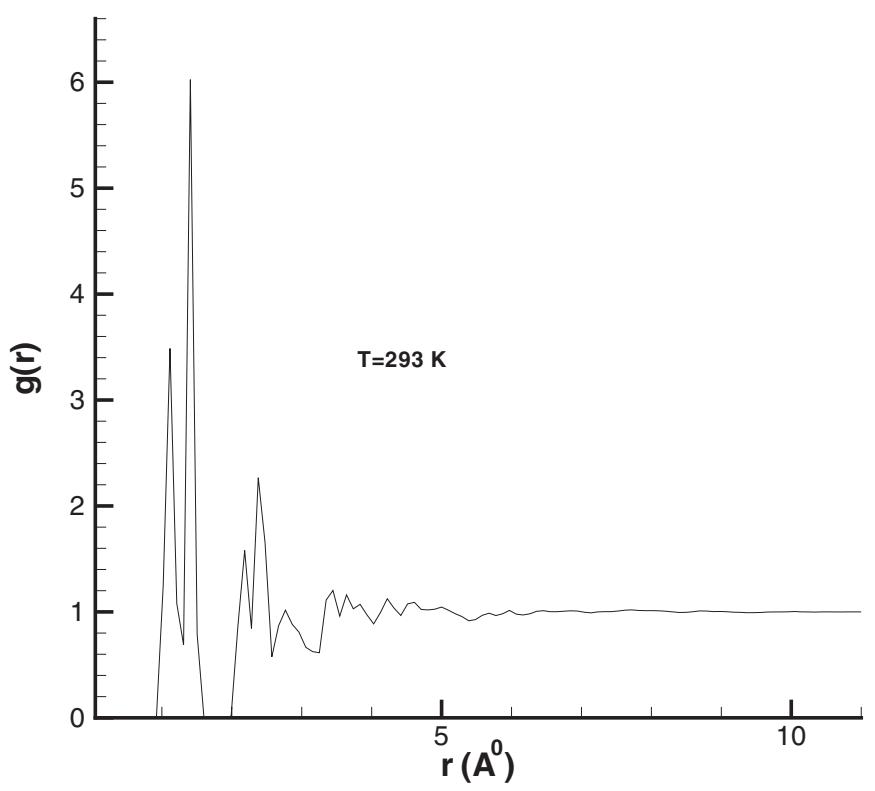

Figure 7. A radial distribution function, $g(r)$ of LARC-SI at $T=293 \mathrm{~K}$.

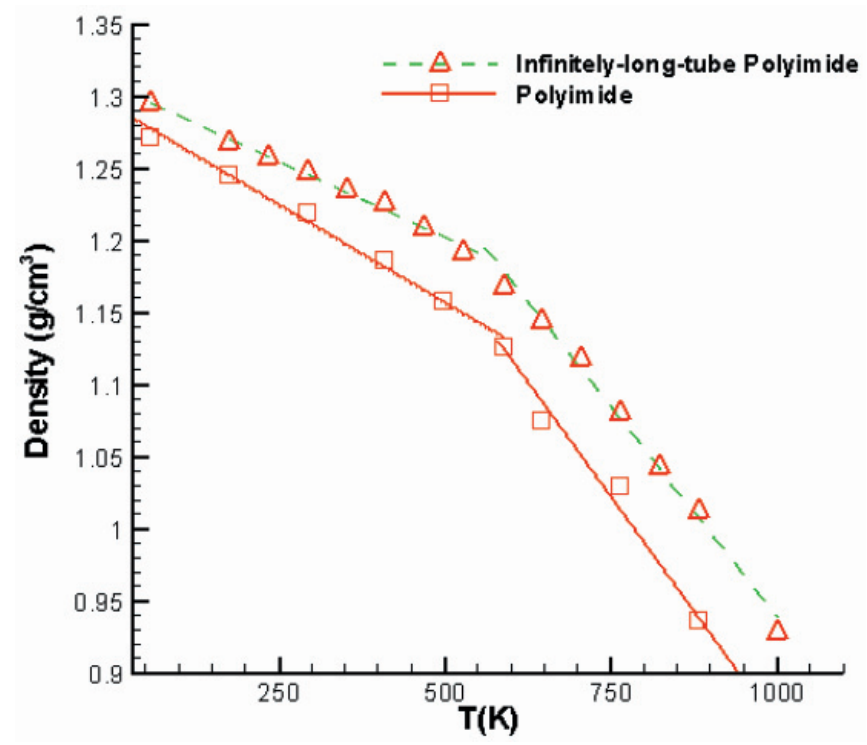

Figure 8. The densities as a function of temperature for the polyimide ( $\square$ ) and the polyimide/infinitely-long-tube composite $(\triangle)$. The curves are divided into two parts, fitted to separate straight lines. The glass transition point is identified by the intersection of the two lines. (This figure is in colour only in the electronic version)

not a problem as long as we use comparable rates when we compare the calculated mechanical properties.

Young's modulus for the isotropic un-reinforced polyimide, obtained by averaging results in the $x$-, $y$ - and $z$-directions, is $E=2.37 \mathrm{GPa}$ at $T=293 \mathrm{~K}$. As the temperature decreases 


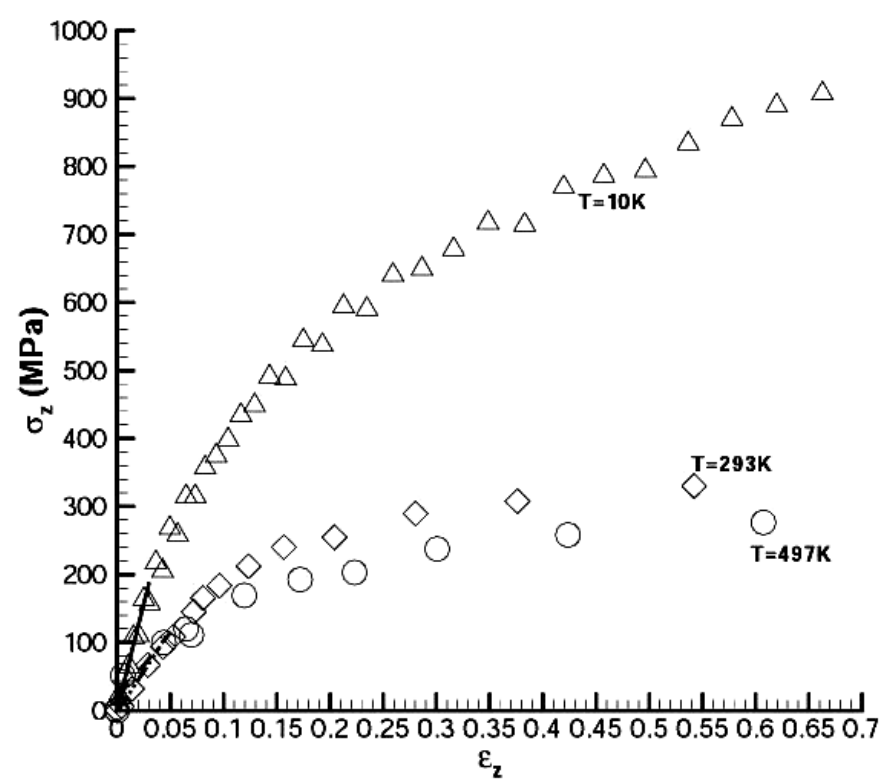

Figure 9. The stress-strain curves in the $z$-direction for un-reinforced polyimide at $T=10 \mathrm{~K}$, $T=293 \mathrm{~K}$ and $T=497 \mathrm{~K}$

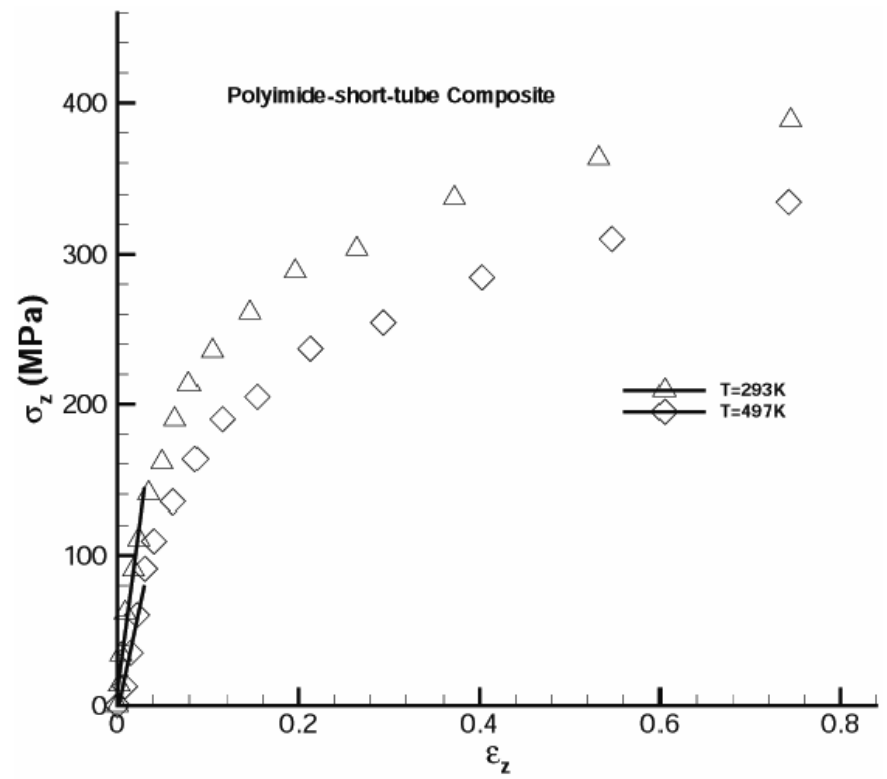

Figure 10. The stress-strain curve for the short-tube composite at $T=293 \mathrm{~K}$ and $T=497 \mathrm{~K}$.

from $T=293 \mathrm{~K}$ to $T=10 \mathrm{~K}$, the Young's modulus increases from $E=2.37 \mathrm{GPa}$ to $E=6.27 \mathrm{GPa}$. When the temperature increases to $T=497 \mathrm{~K}$, the Young's modulus decreases to $E=1.63 \mathrm{GPa}$. The composite becomes stiffer at a lower temperature. In other words, an increase in temperature can soften the composite, as expected. 


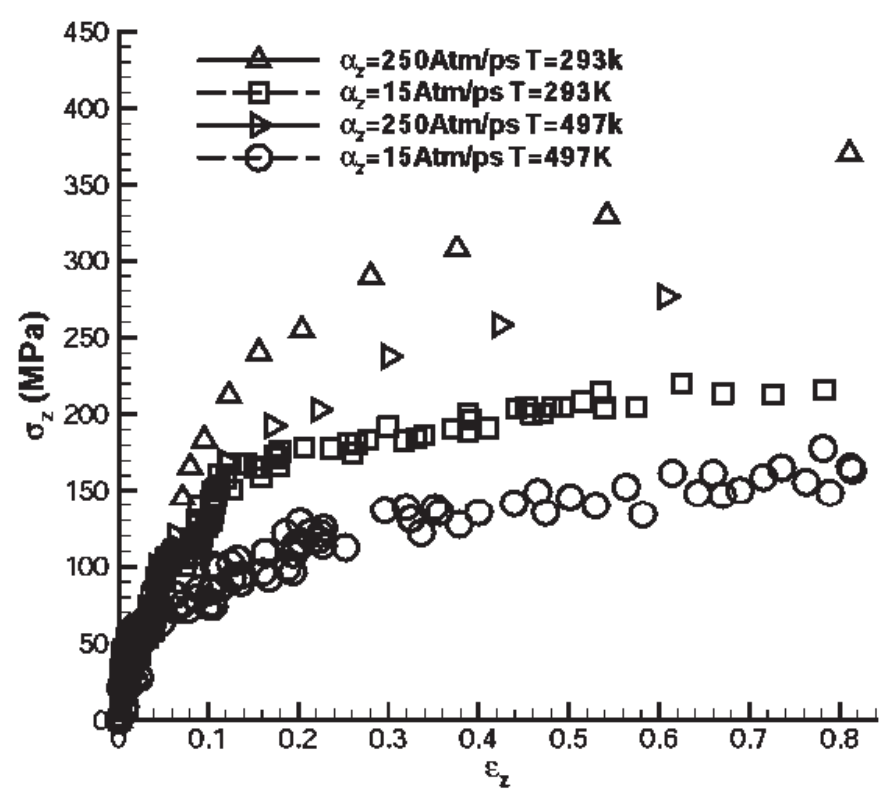

Figure 11. The stress-strain curves at two different tension rates $\alpha_{z}=-250 \mathrm{~atm}(\mathrm{ps})^{-1}$ and $\alpha_{z}=-15 \mathrm{~atm}(\mathrm{ps})^{-1}$ for the polyimide at $T=293 \mathrm{~K}$ and $T=497 \mathrm{~K}$.

The calculated Young's modulus $E=2.37 \mathrm{GPa}$ at $T=293 \mathrm{~K}$ is $36 \%$ lower than the experimental value of $3.7 \mathrm{GPa}$ [24]. We note that the simulation density $\rho=1.21 \mathrm{~g} \mathrm{~cm}^{-3}$ at $T=293 \mathrm{~K}$ was $12 \%$ less than the experimental result, $1.376 \mathrm{~g} \mathrm{~cm}^{-3}$ [7,24]. This is partially attributable to the low polyimide molecular weight in the simulation, which was only 6057 . In the experiments the molecular weight is at least 15880 [24]. In addition, we have neglected Coulomb interactions, which might be expected to make a contribution to the energy due to existence of oxygen and nitrogen atoms in the polymers. We are currently working on a simulation including the effects of Coulomb interactions. Taking these factors into account, the present results for the model polymer appear reasonable. They are closer to the experimental results than those reported by Hu et al using molecular mechanics. Their result for the Young's modulus for polyimide is two times larger than the experimental value [9].

The infinitely-long-tube composite is highly anisotropic due to the carbon NT aligned in the $z$-direction. Stress-strain curves are shown in figure 13 for the $y$-direction and in figure 12 for the $z$-direction. Due to the very high stiffness of the infinitely-long-tube composite, the total time to produce the strain up to $21 \%$ at $T=293 \mathrm{~K}$, in figure 12 , even using a higher tension rate $\alpha_{z}=-250 \mathrm{~atm}(\mathrm{ps})^{-1}$, is $2700 \mathrm{ps}$. This long period in the stretching process dramatically increases the computation time. Also, we found that the fluctuation of the stress and strain was smaller at a higher tension rate than at a lower tension rate. For these two reasons, we use the higher tension rate of $\alpha_{z}=-250 \mathrm{~atm}(\mathrm{ps})^{-1}$ for most of the stretching in this work.

The behaviour of the stress-strain curve in the $y$-direction or the transverse direction for the infinitely-long-tube composite in figure 13 resembles that in figures 9 and 10 . At $T=293 \mathrm{~K}$, the Young's modulus in the transverse direction is $4.12 \mathrm{GPa}$ for the infinitely-longtube composite (compared with 2.37 GPa for the neat polyimide). Although the infinitely-long NT reinforces the modulus in the transverse direction, the effect is not as strong as in the tube direction. The $z$-direction stress-strain curves in the infinitely-long-tube composite in 


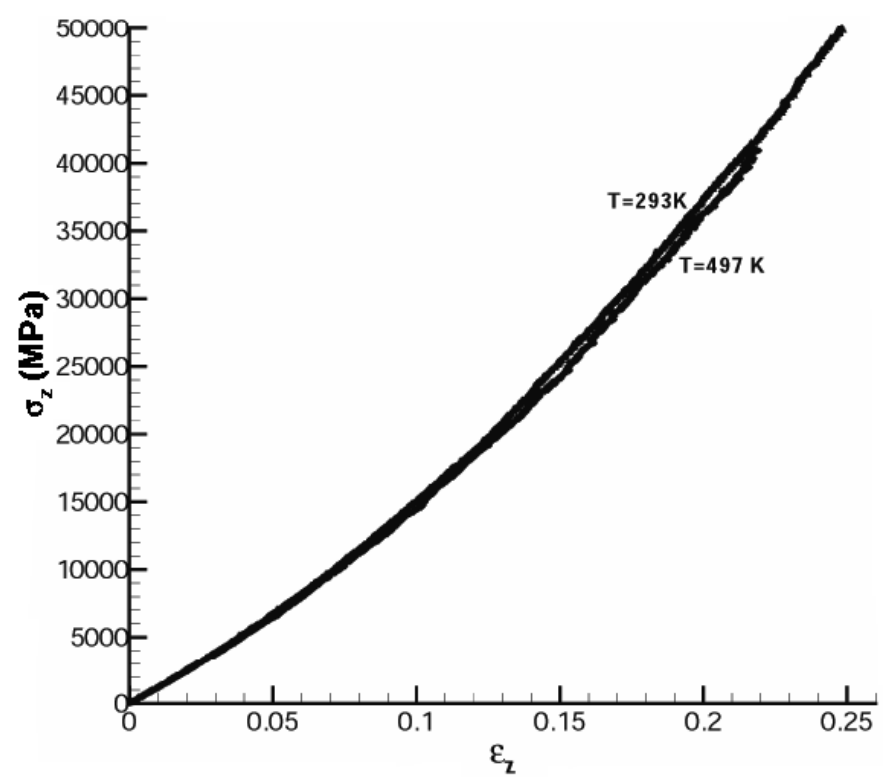

Figure 12. The stress-strain curves in the $z$-direction for infinitely-long-tube composite at $T=293 \mathrm{~K}$ and $T=497 \mathrm{~K}$.

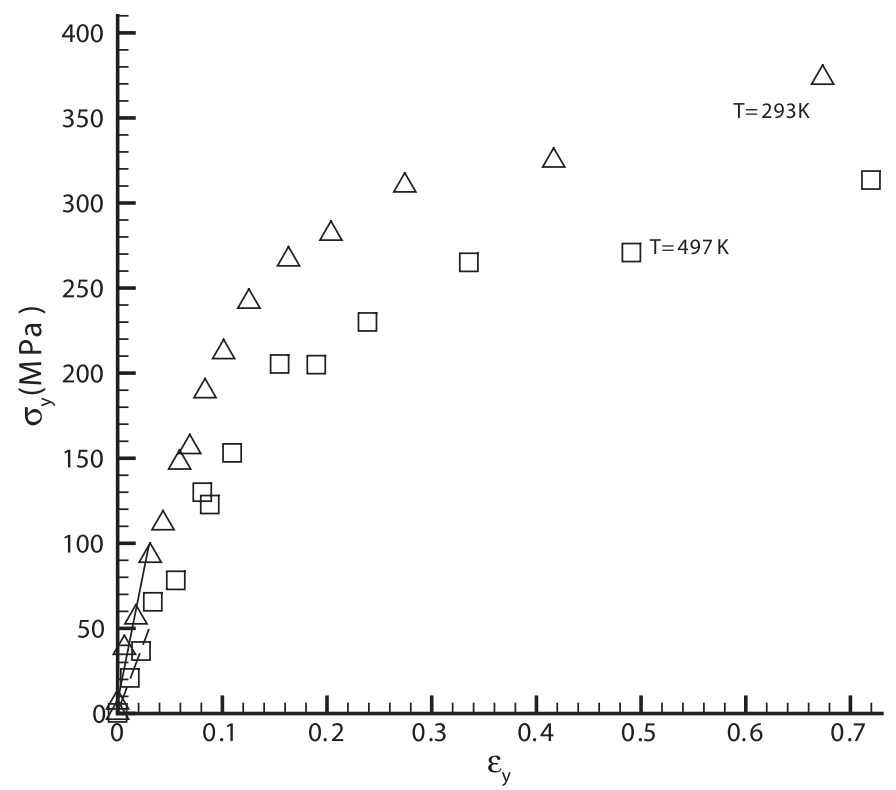

Figure 13. The stress-strain curve in the $y$-direction for the infinitely-long-tube composite.

figure 12 are significantly different from those in the isotropic systems. First, the slope of the linear portion is much larger. The Young's modulus in the tube direction is $135 \mathrm{GPa}$ at $T=293 \mathrm{~K}$ and $129 \mathrm{GPa}$ at $T=497$. These values are 57 and 79 times larger than that of the polyimide without the carbon NT at these two respective temperatures. Second, the shape of the stress-strain curve in the infinitely-long-tube composite is entirely different from that 


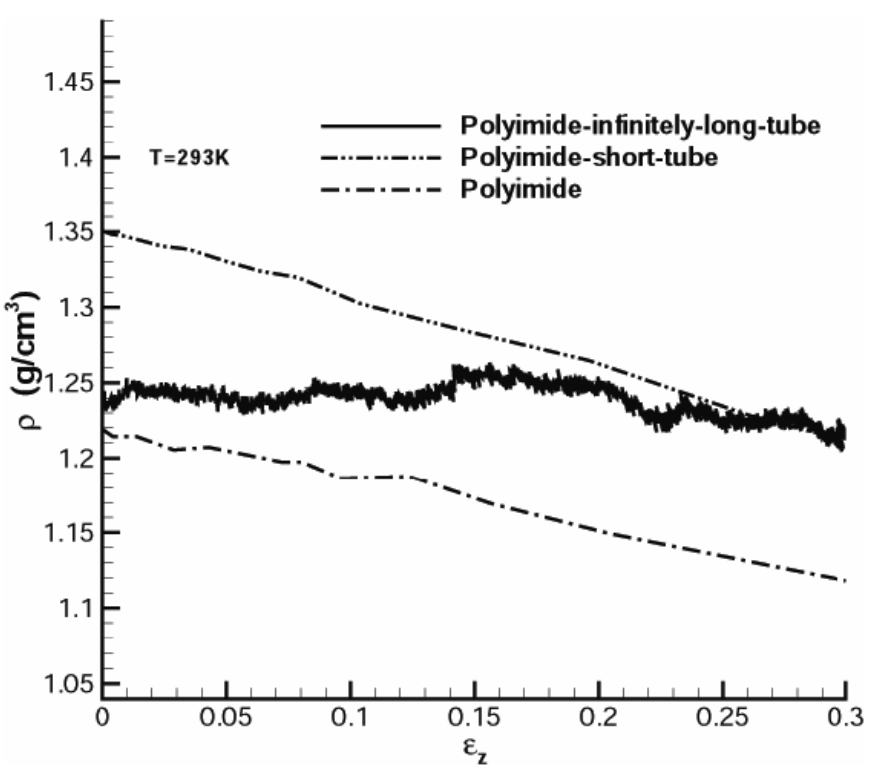

Figure 14. The densities as functions of strain, $\varepsilon_{z}$, for all three different composites during stretching.

in the other two systems studied. The curve is slightly concave for the infinitely-long-tube composite, and no clearly-defined nonlinear portion appears. Third, the temperature softening effect on the Young's modulus is much weaker for the infinitely-long-tube composite than for the neat resin. The Young's modulus decreases only 4\% between 293 and $497 \mathrm{~K}$ (to129 GPa). This is in contrast to the $31 \%$ over the same temperature range for the un-reinforced polyimide.

It must be borne in mind that the situation for an infinitely long NT is entirely different from that for a finitely long NT. Because the tube traverses the simulation box, the tube and the polymer matrix are effectively loaded in parallel. Under these conditions, the modulus of the composite should be describable by a simple rule of mixtures. The longitudinal crystal modulus determined in the validation procedure above can be converted to an effective singletube modulus through simple geometrical factors [25]. Similar considerations allow us to calculate a single-tube density. Assuming the polymer atom/NT carbon distance is $3.4 \AA$, the rule of mixtures for a 16 weight per cent (9.84 volume per cent) composite gives an expected modulus of $134.6 \mathrm{MPa}$, essentially matching the value from the simulation. In contrast, finitelylong tubes depend on load transfer between the matrix polymer and NT. The load transfer will greatly affect the mechanical properties.

For the short-tube composite, the stress-strain curves in the $x$ - and $y$-directions are similar to the $z$-direction curves and are not shown here. The Young's moduli derived from the linear portions of the curves are $3.95 \mathrm{GPa}$ at $T=293 \mathrm{~K}$ and $2.88 \mathrm{GPa}$ at $T=497 \mathrm{~K}, 1.5$ times and 1.4 times larger, respectively, than those in the neat polyimide.

In figure 14 we plot the density as a function of strain, $\varepsilon_{z}$, during stretching for all three systems. It is shown that the density of the polyimide and the short-tube composites decreases as the strains increase, indicating that stretching increases their average atom distance. The density of the infinitely-long-tube composite, on the other hand, stays at the same level (with some fluctuation).

The Poisson ratio is defined by $\lambda_{z y}=-\left(\varepsilon_{y} / \varepsilon_{z}\right)$ and can be estimated during stretching in the $z$-direction since the lengths of the simulation box are variables in the simulations. For the 
polyimide at room temperature, we obtain a Poisson's ratio of 0.35 (average of three directions for this isotropic system). This result is comparable with the experimental result. The Poisson ratio $\lambda_{1}=0.5\left(\lambda_{z x}+\lambda_{z y}\right)=0.59$, averaged from two transverse directions, for the infinitelylong-tube composite. When the composite is stretched in the $x$-direction, it does not contract too much in the $z$-direction due to the very stiff carbon NT in this direction. Thus, the Poisson ratio $\lambda_{2}=0.5\left(\lambda_{x z}+\lambda_{y z}\right)=0.0083$ is much smaller than $\lambda_{3}=0.5\left(\lambda_{x y}+\lambda_{y x}\right)=0.65$.

Finally we warn that in real experiments the carbon NT is not infinitely long and the Young's modulus should be much lower than our simulation results due to the limitation of stress transfer. The Young's modulus increase by 57 times in the NT direction for the infinitelylong NT composite will never be observed in experiments. Our simulation results are for a theoretically ideal case.

\section{Conclusion}

An aromatic polyimide and its mixtures with carbon NT are prepared in a computer using MD simulation, repeated energy minimization and cooling processes. The stress-strain curves, Young's modulus, Poisson ratio and volumes are computed at different temperatures using a constant tension algorithm in MD simulations. We find through the density-temperature curves that the glass transition temperature is $570 \mathrm{~K}$ for the model polyimide and $550 \mathrm{~K}$ for the polyimide-infinitely-long-tube composite. The Young's modulus in the tube direction is increased 57 times by adding an infinitely long carbon NT of $16 \%$ composite by weight while the Young's modulus in the transverse direction is increased 1.73 times. By adding short carbon NTs at $36 \%$ by weight the Young's modulus is increased 1.67 times at the room temperature. We also demonstrate that carbon NTs can reduce the softening effect of temperature on mechanical properties and increase the ability to resist deformation.

\section{Acknowledgments}

D Qi appreciates the hospitality from National Institute of Aerospace and Structures and Materials Competency, NASA Langley Research Center during his visit in 2003. A part of this work was conducted during this period. Useful discussions with Drs T Gates, S J V Frankland, G M Odegard, K Wise and C Park are acknowledged. Computational time allocation on Cray T3E at the Pittsburgh Supercomputing Center through National Computational Science Alliance is appreciated.

\section{References}

[1] Shepherd P D, Golemba F J and Maine F W 1974 Adv. Chem. Ser. 13441

[2] Kojima Y, Usuki A, Kawasmi M, Okada A, Fukushima Y, Kuraucji T and Kamigaito O 1993 J. Mater Res. 81185

[3] Overney G, Zhong W and Tomanek D 1993 Z. Phys. D: At. Mol. Clusters 2793

[4] Meyyappan M and Han J 1998 Prototype Tech. Int. 614

[5] Park C, Ounaies Z, Watson K A, Pawlowski K, Lowther S E, Connell J W, Siochi E J, Harrison J S and St Clair T L Polymer-single wall carbon nanotube composites for potential spacecraft applications, NASA/CR-2002211940 ICASE Report No 2002-36

[6] Frankland S J V, Harik V M, Odegard G M, Brenner D W and Gates T S 2003 The stress-strain behavior of polymer-nanotube composites from molecular dynamics simulation Compos. Sci. Technol. 63 1655-61

[7] Bryant R G 1996 LARC-SI: a soluble aromatic polyimide High Perform. Polym. 8 607-15

[8] Hou T H and Bryant R G 1997 Processing and properties of IM7/LARC-SI polyimide composites High Perform. Polym. 9 437-48 
[9] Fan C F and Hsu S L 1991 Application of the molecular simulation technique to generate the structure of an aromatic polysulfone system Macromolecules 24 6244-9

[10] Plimpton S J 1995 Fast parallel algorithms for short-range molecular dynamics J. Comp. Phys. 117 1-19

[11] Clark M, Cramer R D C and Opedenbosch N V 1989 Validation of the general purpose Tripos 5.2 force field J. Comp. Chem. $10982-1012$

[12] Pinel E, Brown D, Bas C, Mercier R, Alberola N D and Neyertz S 2002 Chemical influence of the dianhydride and diamine structure on a series of copolyimides studies by molecular dynamics simulations Macromolecules 35 10198-200

[13] White C T, Robertson D H and Mintmire J W 1993 Helical and rotational symmetries of nanoscale graphitic tubules Phys. Rev. B 47 5485-8

[14] Nose S 1984 Mol. Phys. 52255

[15] Hoover W G 1985 Canonical dynamics: equilibrium phase-space/distributions Phys. Rev. A 31 1695-7

[16] Brown D and Clarke J H R 1998 Molecular dynamics simulation of an amorphous polymer under tension. I. Phenomenology Macromolecules 24 2075-82

[17] Ray J R 1988 Elastic constants and statistical ensembles in molecular dynamics Comput. Phys. Rep. 8 109-52

[18] Treacy M M J, Ebbsen T W and Gibson J M 1996 Nature (London) 382678

[19] Hernandez E and Subbaswamy K R 1998 Phys. Rev. Lett. 804502

[20] Popov V N, Doren V E V and Balkanski M 2000 Elastic properties of crystals of single-walled carbon nanotubes Solid State Commun. 114 395-9

[21] Theodorous D N and Suter U W 1986 Atomistic modeling of mechanical properties of polymeric glasses Macromolecules 19 139-54

[22] Fan C F and Hsu S L 1992 Application of the molecular simulation technique to characterize the structure and properties of an aromatic polysulfone system. 2. Mechanical thermal properties Macromolecules 25 266-70

[23] Fan C F, Cagin T and Chen Z M 1994 1. Force field, static structure and mechanical properties Macromolecules 27 2383-91

[24] Lee M N, Whitley K S and Gates T S 2002 The role of molecular weight and temperature on the elastic and viscoelastic properties of a glassy thermoplastic polyimide Int. J. Fatigue 24 185-95

[25] Pipes R B, Frankland S J V, Hubert P and Saether E 2003 Self-consistent properties of carbon nanotubes and hexagonal arrays as composite reinforcements Compos. Sci. Technol. 63 1348-58 